\title{
Exosomes from HPV-16 E7-pulsed dendritic cells prevent the migration, M1 polarization, and inflammation of macrophages in cervical cancer by regulating catalase 2 (CAT2)
}

\author{
Guangping Zhang ${ }^{1}$, Yanan Liao ${ }^{1}$, Xiaoping Pan $^{2}$, Xiaoli Zhang ${ }^{3}$ \\ ${ }^{1}$ Department of Gynecology, Affiliated Huadu Hospital, Southern Medical University (People’s Hospital of Huadu District), Guangzhou, \\ China; ${ }^{2}$ Clinical Laboratory, Affiliated Huadu Hospital, Southern Medical University (People's Hospital of Huadu District), Guangzhou, China; \\ ${ }^{3}$ Department of Obstetrics, Affiliated Huadu Hospital, Southern Medical University (People's Hospital of Huadu District), Guangzhou, China \\ Contributions: (I) Conception and design: G Zhang; (II) Administrative support: X Zhang, G Zhang; (III) Provision of study materials or patients: G \\ Zhang, Y Liao; (IV) Collection and assembly of data: X Pan; (V) Data analysis and interpretation: X Zhang, G Zhang; (VI) Manuscript writing: All \\ authors; (VII) Final approval of manuscript: All authors. \\ Correspondence to: Xiaoli Zhang. Department of Obstetrics, Affiliated Huadu Hospital, Southern Medical University (People's Hospital of Huadu \\ District), 48 Xinhua Road, Huadu District, Guangzhou 510800, China. Email: ogzhxl@163.com.
}

Background: Cervical cancer is mainly caused by persistent infection with human papillomavirus (HPV), especially HPV-16. Recently, HPV-16 E7-modified dendritic cells (DCs) have been reported to play a blocking role in the progression of cervical cancer. Conversely, the effect and mechanism of HPV-16 E7pulsed DCs in cervical cancer are not entirely clear.

Methods: DCs from the peripheral blood of patients with cervical cancer were induced with lipopolysaccharide and identified through the detection of cluster of differentiation (CD)11c, major histocompatibility complex (MHC)-II, CD83, and CD40 levels, and exosomes from HPV-16 E7-pulsed and catalase 2 (CAT2)-silenced DCs were extracted and identified through transmission electron microscopy and the detection of markers. Additionally, the migration, inflammatory factors, and polarization of macrophages were confirmed using Transwell, enzyme-linked immunoassay, and Western blot of arginase-1 (Arg-1) and inducible nitric oxide synthase (iNOS). In vivo, we also built a mice xenograft model of HPV cervical cancer. Results: We first successfully induced and identified DCs from cervical cancer patients, and successfully extracted and confirmed the exosomes from the constructed HPV-16 E7-pulsed and CAT2-silenced DCs. Subsequently, we proved that exosomes from HPV-16 E7-pulsed DCs restrained migration and inflammation and induced M2 polarization in macrophages, while the effect of exosomes from CAT2-silenced DCs on macrophage migration, polarization, and inflammation was opposite to that of exosomes from HPV-16 E7pulsed DCs, and the 2 affected each other. Additionally, we found that exosomes from CAT2-silenced DCs also prevented growth and M2 polarization in a mice xenograft model of HPV cervical cancer.

Conclusions: Exosomes from HPV-16 E7-pulsed DCs blocked cervical cancer progression by regulating macrophage function, and its mechanism was relevant to CAT2.

Keywords: Cervical cancer; exosomes; HPV-16 E7 (HPV16E7); dendritic cells (DCs); macrophages

Submitted Nov 25, 2021. Accepted for publication Feb 22, 2022.

doi: 10.21037/atm-21-6998

View this article at: https://dx.doi.org/10.21037/atm-21-6998 


\section{Introduction}

Cervical cancer is the $4^{\text {th }}$ most common carcinogenesis in women worldwide, with about $85 \%$ of total new cases worldwide originating in developing countries (1). In 2018, there were 570,000 cases of and 311,000 deaths from cervical cancer worldwide (2). Advanced cervical cancer has a low cure rate and a high recurrence rate (3). Additionally, as the tumor size of advanced cervical cancer is large, immune tolerance develops easily during chemotherapy (4). Thus, an effective therapy is needed to reduce tumor recurrence and metastasis. The persistent infection of high-risk human papillomavirus (HR-HPV) has been reported to be the direct cause of cervical intraepithelial neoplasia and cervical cancer $(5,6)$. Of the HR-HPV types, HPV-16 has the highest incidence, and accounts for $53 \%$ of all cervical cancer cases (7). HPV-16 E7 (HPV16E7), which is a key molecule in HPV carcinogenesis, is only selectively expressed in tumor cells (8). E7 protein is an ideal target protein for cervical cancer therapy (8). Thus, further investigations into the function and mechanism of HPV16E7 in cervical cancer might provide a theoretical basis for cervical cancer therapy.

Dendritic cells (DCs), the strongest antigen-presenting cells (APCs), are also the only APCs that can activate primary $\mathrm{T}$ cells (9). Mature DCs (mDCs) can induce the generation of cytotoxic $\mathrm{T}$ lymphocytes (CTLs) by presenting antigens to $\mathrm{T}$ lymphocytes, which can activate specific immunity, and dissolve and kill tumor cells (10). As far as we know, DCs with normal function can activate the immune function of the body and resist tumorigenesis (11). A new study shows that exogenous modified DCs can stimulate the proliferation of cytotoxic $\mathrm{T}$ cells, thus triggering the body's anti-cancer immune function (11). However, the antitumor of DCs was remain unclear. Nowadays, researchers are focusing on the regulatory mechanism of tumorigenesis caused by DCs exocrine. For example, recent research suggests that a modified DCs exert an enhanced anti-cervical cancer immunity by inducing DC-derived exosomes (12). Thus, researchers are interested in how to best transform differentiated conventional DCs into mature and activated DCs (13). At present, the main forms of DC vaccine preparation include tumor specific antigens, gene-modified DCs, tumor nucleic acid sensitized DCs, and the fusion of tumor cells and DCs (14). Among these, gene-modified DCs can prevent the immune escape of tumors and greatly enhance the antigen presentation of DCs, and thus have been widely applied in research (15).

Vaccines against HPV infection mainly comprised prophylactic and therapeutic vaccines (16). Prophylactic vaccines mainly produce neutralizing antibodies to block native virus particles (17). Therapeutic vaccines mainly induce a specific CTL response to HPV (18). For example, cancer antigens extracted from cervical cancer cells are loaded with autologous DCs from patients to make vaccines, and tumor cells are then co-cultured with the DC vaccine, and its CTLs have a powerful lethality and cause a specific immune response against cervical cancer cells (19). It has been reported that HPV16E7-pulsed DCs can activate cellular immunity and induce the regulatory function of killing tumor cells (20). Also in cervical cancer, HPV16E7-pulsed DCs cause a strong cytolytic activity of T lymphocytes, resulting in lysing naturally HPV-16-infected tumor cells (21). Immunotherapy could adopt this therapeutic vaccine, which prevents tumor recurrence and spread by stimulating the cellular immunity of patients to kill and eliminate tumor cells and epithelial cells infected with HPV.

Tumor-associated macrophages (TAMs) refer to macrophages that infiltrate and affect the development of tumors during tumorigenesis (22). TAMs have the phenotype of M2 macrophages, and can express cluster of differentiation (CD)68 and CD163 (23). Researchers discovered that high TAMs are associated with a poor prognosis and affect the occurrence, development, and metastasis of various solid tumors, including cervical cancer tumors $(24,25)$. However, the association between HPV16E7 expression and TAMs in cervical cancer is not completely clear.

In this study, a recombinant adenovirus vector carrying the HPV16E7 gene was used to infect DCs from cervical cancer patients or mice to prepare the DC vaccine. Next, we observed the effect of HPV16E7-pulsed DCs on macrophage migration and polarization by up-taking exosome from DCs for the first time. The findings of the current study provide a basis for the immunotherapy of cervical cancer, and thus are of important practical significance.

We present the following article in accordance with the ARRIVE reporting checklist (available at https://atm. amegroups.com/article/view/10.21037/atm-21-6998/rc).

\section{Methods}

\section{Extraction of DCs from cervical cancer patients}

Peripheral blood $(20 \mathrm{~mL})$ was extracted from cervical cancer patients, who had been admitted to the People's Hospital 
of Huadu District in 2019, and heparin was applied for anticoagulant. After centrifugation, the upper plasma was placed in a $56-^{\circ} \mathrm{C}$ water bath for $30 \mathrm{~min}$, and collected for standby application by centrifugation after standing at $4{ }^{\circ} \mathrm{C}$ for $15 \mathrm{~min}$. Dulbecco's phosphate-buffered saline (PBS) was added to the centrifuged cell precipitates, and then the mixture was slowly transferred to a centrifuge tube containing $10 \mathrm{~mL}$ of lymphocyte separation medium. After centrifugation, the cells of the white membrane layers were suspended with Alys-505 medium containing $10 \%$ plasma. The cells $\left(5 \times 10^{6}\right.$ cells $\left./ \mathrm{mL}\right)$ were inoculated into a 6-well plate and cultured for $2 \mathrm{~h}$ at $37^{\circ} \mathrm{C}$ with $5 \%$ carbon dioxide $\left(\mathrm{CO}_{2}\right)$. Next, the cells were cultured in Alys-505 medium with $100 \mathrm{ng} / \mathrm{mL}$ of granulocyte-macrophage colonystimulating factor (GM-CSF), $10 \mathrm{ng} / \mathrm{mL}$ of interleukin (IL)-4, and $10 \%$ plasma. The collected DCs were then induced by $200 \mathrm{ng} / \mathrm{mL}$ of lipopolysaccharide (LPS), and the morphology of the mDCs was observed under a light microscope. The present study was approved by the Ethics Committee of the People's Hospital of Huadu District and conducted in accordance with the Declaration of Helsinki (as revised in 2013). Informed consent was granted an exemption by the hospital.

\section{Imтипоfluorescence (IF) staining}

The DCs were first seeded on a cover slide in a 6-well plate, and then fixed with $4 \%$ paraformaldehyde permeated with $0.5 \%$ Triton $\mathrm{X}-100$. After washing, the DCs were sealed with $1 \%$ bovine serum albumin (BSA), anti-CD11c (Abcam, Cambridge, UK) at $4{ }^{\circ} \mathrm{C}$ overnight, and then incubated with fluorescence secondary antibody (Abcam, Cambridge, UK) for $1 \mathrm{~h}$. Then, 4',6-diamidino-2-phenylindole- $2 \mathrm{HCl}$ was dripped onto the DCs for $5 \mathrm{~min}$, and images were taken under a fluorescence microscope.

\section{Flow cytometry}

The cultured DCs were collected and adjusted to $1 \times 10^{7} / \mathrm{mL}$ with a cold buffer (pH $7.2 \mathrm{PBS}+150 \mathrm{mM} \mathrm{NaCl}+$ $\left.0.09 \% \mathrm{NaN}_{3}+0.2 \% \mathrm{BSA}\right)$. Twenty $\mu \mathrm{L}$ of fluorescein isothiocyanate (FITC)-CD83, $20 \mu \mathrm{L}$ of phycoerythrin (PE)$\mathrm{CD} 80$, or $20 \mu \mathrm{L} \mathrm{PE}-\mathrm{CD} 40$ was added to each $100 \mu \mathrm{L}$ cell of suspension, which was kept at $4^{\circ} \mathrm{C}$ for $30 \mathrm{~min}$ away from light. Twenty $\mu \mathrm{L}$ of FITC-IgG or $20 \mu \mathrm{L}$ of PE-IgG was added to the control group. After washing, the DCs were suspended with $250 \mu \mathrm{L}$ of PBS, and the DC phenotype was tested by flow cytometry.

\section{Extraction of DCs from mice}

C57BL/6 male mice (aged 4-5 weeks) were purchased from Animal Experiment Center, Southern Medical University and kept in a specific pathogen-free (SPF) animal house under standard feeding conditions. All animal experiments were performed and approved by the Institutional Animal Care and Use Committee (IACUC) of the Affiliated Huadu Hospital, Southern Medical University (also named People's Hospital of Huadu District), in compliance with the Affiliated Huadu Hospital of Southern Medical University guidelines for the care and use of animals. The C57BL/6 mice were sacrificed, and the femur, tibia, and muscle tissue were removed under sterile conditions. The marrow was obtained by rinsing the marrow cavity with PBS. After centrifugation, the cells were collected and $4 \mathrm{~mL}$ of erythrocyte lysate was added. After standing, the cell density medium was adjusted to $3 \times 10^{9}$ cells $/ \mathrm{mL}$. The cells were evenly placed in 6-well plates, and $10 \mathrm{ng} / \mathrm{mL}$ of recombinant mouse GM-CSF (rmGM-CSF) and $10 \mathrm{ng} / \mathrm{mL}$ of recombinant mouse IL-4 (rmIL-4) was added, and the cells were then cultured at $37^{\circ} \mathrm{C}$ with $5 \% \mathrm{CO}_{2}$.

\section{Construction and infection of HPV16E7 adenovirus}

Adenovirus vectors [pAdTrack-CMV and pET-32a(+)-E7] were digested by BgIII and HindIII. The target gene and the shuttle plasmid (pAdTrack-CMV) were then ligated by double enzyme digestion and transformed into E. coli DH5. The recombinant plasmid was identified by polymerase chain reaction (PCR), and confirmed by sequencing. Next, the pAdTrack-CMV-E7 plasmid was transformed into E. coli BJ5183 containing adenovirus genome plasmid (AdEasy), and identified by sequencing. The HEK293 cells were cotransfected with the adenovirus plasmids for packaging using Lipofectamine $^{\mathrm{TM}} 3000$ (Invitrogen) for $48 \mathrm{~h}$. The HEK293 cells were massively infected with recombinant adenovirus, and the pathological cells were collected. After repeated freezing and thawing, the supernatant was collected and the virus titer was tested by cytopathic assay (CCID50). The DCs were infected with 200 multiplicity of infection (MOI) recombinant adenovirus (pAd-E7). After $2 \mathrm{~h}$, the original medium was replaced with medium, including $5 \mathrm{ng} / \mathrm{mL}$ of $\mathrm{rmGM}-\mathrm{CSF}$ and $5 \mathrm{ng} / \mathrm{mL}$ of rmIL-4.

\section{Cell transfection and treatment}

Catalase 2 (CAT2) small-inhibitory ribonucleic acids (siRNAs) 
$\# 1, \# 2, \# 3$ and the negative control (NC) were purchased from GenePharma (Suzhou, China), and the DCs were transfected with si-CAT2 and si-NC using Lipofectamine 3000 (Invitrogen) for $4 \mathrm{~h}$. The CAT2-silenced DCs were then harvested. Additionally, HPV16E7-pulsed or/and CAT2-silenced DCs were processed with exosome inhibitor (GW4869). The HPV16E7-pulsed and CAT2-silenced DCs were co-cultured with $\mathrm{M} 0$ macrophages at $37^{\circ} \mathrm{C}$.

\section{Exosome extraction from $\mathrm{DCs}$}

We first collected the supernatant of HPV16E7-pulsed and CAT2-silenced DCs. After centrifugation, the floating cells were removed and the precipitation was discarded. After further centrifugation, the dead cells and exfoliated vesicles were removed. Next, the precipitation was collected and resuspended after centrifugation $(140,000 \times \mathrm{g}$ for $70 \mathrm{~min})$.

\section{Transmission electron microscope (TEM)}

The exosomes from DCs $(10 \mu \mathrm{L})$ were diluted with $10 \mu \mathrm{L}$ of PBS, and then dropped onto $2 \mathrm{~mm}$ copper mesh at room temperature for $1 \mathrm{~min}$. Next, the excess liquid was sucked up with filter paper, and the exosomes were re-stained with 3\% (W/V) sodium phosphotungstate for $1 \mathrm{~min}$. After washing, the exosomes were observed and photographed through TEM (JEM-1011, JEOL, Japan).

\section{ELISA assay}

The cultured mDCs in each group were centrifugally collected, and IL-10, transforming growth factor beta (TGF- $\beta$ ), tumor necrosis factor alpha (TNF- $\alpha$ ), and IL-12 levels were confirmed using enzyme-linked immunoassay (ELISA) kits as per the instructions. These kits included the IL-10 ELISA kit (Ray Biotech, Norcross, USA), TGF- $\beta$ ELISA kit (R\&D Systems, Minnesota, USA), TNF- $\alpha$ ELISA kit (Abcam, Cambridge, UK, ab46087), and IL-12 ELISA kit (BD Bioscience, New Jersey, USA).

\section{RT-qPCR assay}

We harvested total RNAs from groups of tumors with TRIzol reagent (Invitrogen, MA, USA). The total RNAs were then reversed using the PrimeScript ${ }^{\mathrm{TM}} \mathrm{RT}$ Reagent Kit (Takara, Tokyo, Japan). Subsequently, SYBR Green qPCR master Mix (DBI Bioscience, Ludwigshafen, Germany) was used to monitor the levels of arginase-1 (Arg-1), inducible nitric oxide synthase (iNOS), and CAT2. The relative levels were confirmed by the $2^{-\Delta \Delta \mathrm{Ct}}$ method.

\section{Western blot assay}

The treated exosomes or macrophages were lysed with radioimmunoprecipitation assay lysis buffer (Beyotime, Shanghai, China), and total protein was extracted after centrifugation. After the quantitative analysis, the protein in the appropriate loading buffer was denaturated by boiling at $100{ }^{\circ} \mathrm{C}$ for $10 \mathrm{~min}$. Next, the proteins were separated with sodium dodecyl sulfate polyacrylamide gel electrophoresis, and transferred to polyvinylidene fluoride membranes. After sealing, anti-TSG101 (Abcam, Cambridge, UK), anti-CD81 (Abcam, Cambridge, UK), anti-Arg-1 (Abcam, Cambridge, UK), and anti-iNOS (Abcam, Cambridge, UK) were added to the membranes that were kept at $4{ }^{\circ} \mathrm{C}$ overnight. The membrane was then washed and exposed to secondary antibodies for $1 \mathrm{~h}$. After ECL coloring, each band was exposed and developed.

\section{Transwell assay}

The macrophages ( $400 \mu \mathrm{L}$ with $1 \times 10^{5}$ cells) were inoculated into the upper chamber of the Transwell plates, and $600 \mu \mathrm{L}$ of complete medium containing $10 \mathrm{nmol} / \mathrm{L}$ of $\mathrm{C} 5$ a was added into the lower chamber. After incubation for $2 \mathrm{~h}$ at $37{ }^{\circ} \mathrm{C}$ with $5 \% \mathrm{CO}_{2}$, the serum-free medium in the upper chamber was removed, and the lower cells were fixed with $4 \%$ paraformaldehyde for $10 \mathrm{~min}$. The unmigrated cells were gently wiped with a cotton swab. After staining, we randomly selected 5 fields under an inverted microscope to count the migrated cells.

\section{Statistical analysis}

All the experiments in the current research were repeated 3 times. All the data are presented as mean \pm standard deviation, and were analyzed by SPSS 20.0 (SPSS, Inc., Chicago, USA). Comparisons between groups were conducted by a one-way analysis of variance. A P value $<0.05$ was considered statistically significant.

\section{Results}

\section{Induction and identification of DCs}

DC-based immunotherapy is the focus of cancer research (26). To confirm the possible action of DCs 

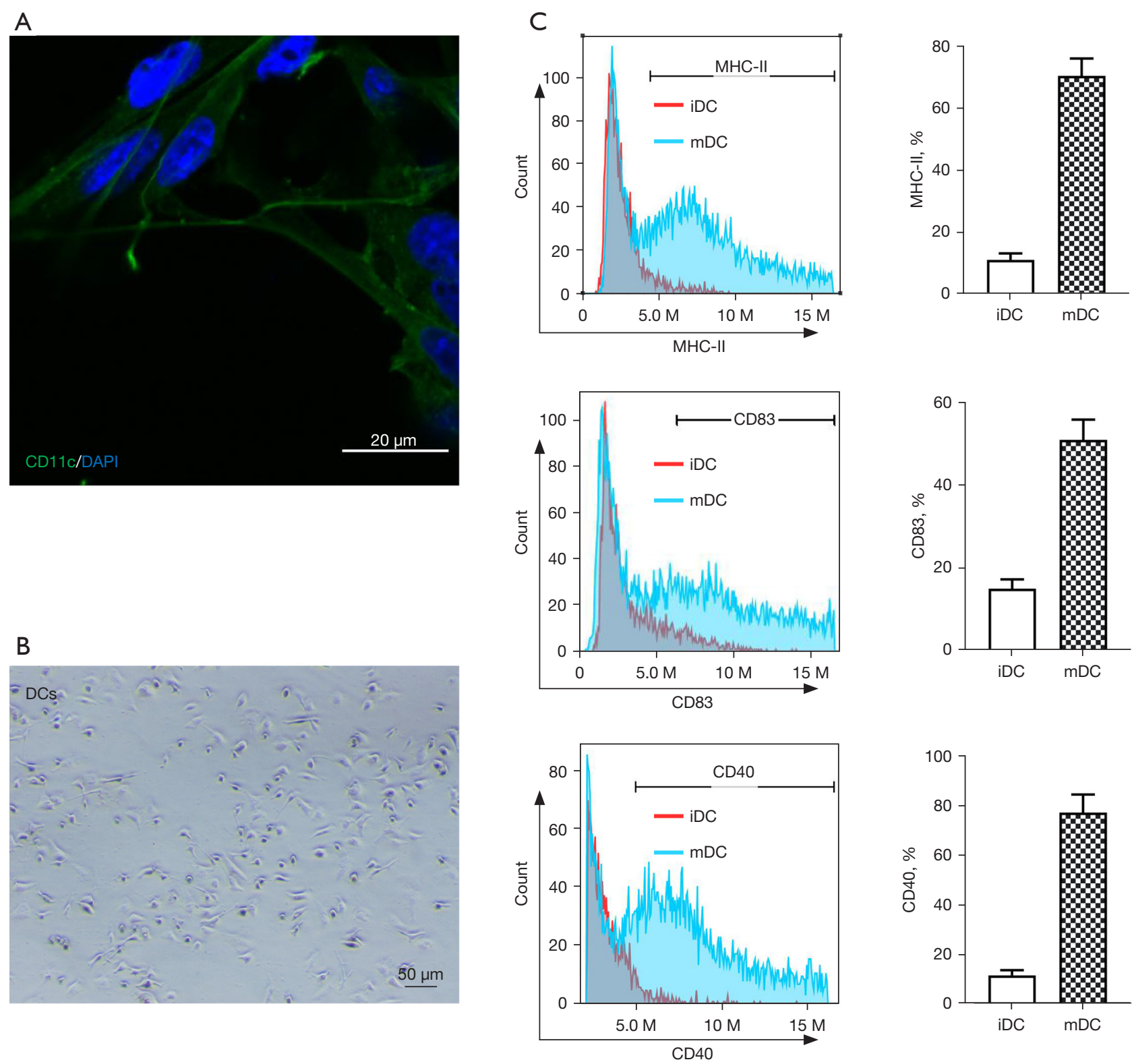

Figure 1 Induction and identification of DCs. (A) CD11c expression was monitored through IF staining in induced DCs. Magnification, 400x. (B) The morphology of the induced DCs was observed with a light microscopy. Magnification, 100x. (C) Flow cytometry was used to determine the expressions of MHC-II, CD83, and CD40 on iDC and mDC surface. iDC, immature dendritic cells; mDC, mature dendritic cells; DCs, dendritic cells; IF, immunofluorescence.

in cervical cancer, we first extracted peripheral blood mononuclear cells (PBMCs) from patients with cervical cancer, and cultured the DCs in the condition of IL-4 and GM-CSF. The DCs were then induced to mature through LPS treatment after 6 days. First, IF staining results signified that the DCs' surface molecules (CD11c) were positively expressed, indicating the successful separation of
DCs (see Figure 1A). Simultaneously, we observed that the boundaries of the DCs had a dendritic spinous appearance under a light microscope (see Figure 1B). Additionally, the expressions of MHC-II, CD83, and CD40 were more heightened on the mDCs surfaces than the immature DCs (iDC) surfaces (see Figure 1C). Thus, the data indicated that we had successfully induced DCs, which came from PBMCs 

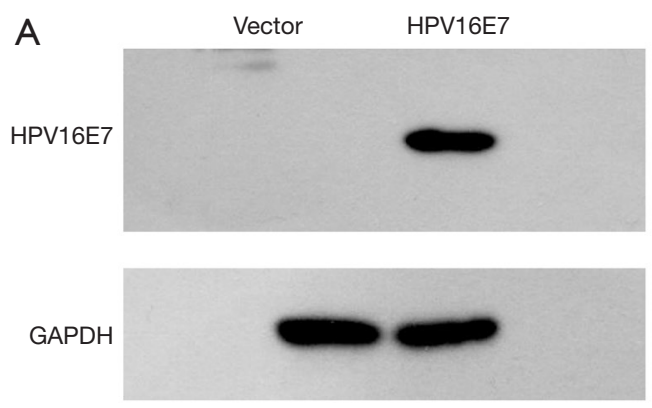

B

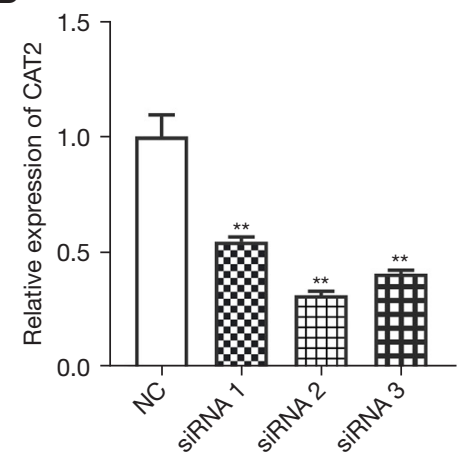

C

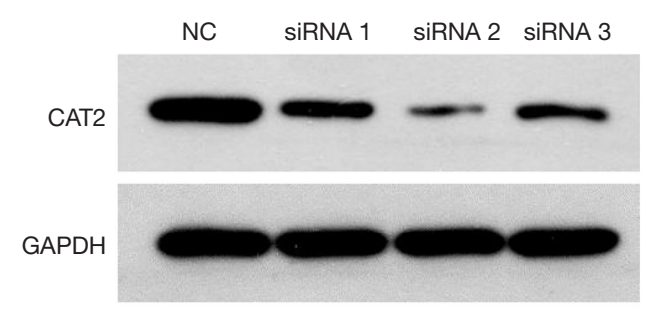

Figure 2 HPV16E7 was overexpressed and CAT2 was silenced in DCs. (A) HPV16E7 expression was tested using Western blot in DCs infected with HPV16E7 adenovirus. DCs were transfected with siRNA\#1, \#2, and \#3 targeting CAT2, and then CAT2 expression was confirmed with RT-qPCR (B) and Western blot (C). **, $\mathrm{P}<0.01$, vs. NC. CAT2, catalase 2; HPV16E7, human papillomavirus type 16 E7; DCs, dendritic cells; NC, negative control.

in patients with cervical cancer.

\section{HPV16E7 was overexpressed and CAT2 was silenced in DCs}

To further verify the effects of HPV16E7-pulsed DCs on cervical cancer progression, we first overexpressed HPV16E7 in DCs. As Figure $2 A$ shows, HPV16E7 expression was notably more elevated in the HPV16E7 overexpression group than the vector group in the induced DCs. Additionally, we also silenced CAT2 in DCs through transfection with CAT2 siRNA\#1, \#2, and \#3. The mRNA and protein levels of CAT2 were significantly lower in the HPV16E7 overexpression group than the vector group (see Figure 2B,2C). Generally, these results indicated that we successfully overexpressed HPV16E7 and silenced CAT2 in DCs via transfection.

\section{Exosomes from HPV16E7-pulsed and/or CAT2-silenced DCs regulated macrophage differentiation and migration}

Subsequently, we further isolated exosomes from the
DCs that had overexpressed HPV16E7 and/or silenced CAT2. Under a TEM, it was clearly observed that the exosomes extracted from the DCs in each group were double-membrane-like structures, circular or quasicircular, with a diameter of 30-100 nm, relatively uniform in size and uniform in distribution (see Figure $3 A$ ). The Western blotting data showed that the exosome marker proteins (TSG101 and CD81) were highly expressed in the extracted exosomes of each group, indicating that the extracted exosomes were of high purity (see Figure $3 B$ ). Next, we used these exosomes to treat M0 macrophages. First, the Transwell data revealed that the migration ability of macrophages was observably suppressed by exosomes from HPV16E7-pulsed DCs, or induced by exosomes from CAT2-silenced DCs. Additionally, exosomes from HPV16E7-pulsed DCs reversed the enhancement of macrophage migration mediated by exosomes from CAT2silenced DCs, and exosomes from CAT2-silenced DCs also reversed the suppression of macrophage migration mediated by exosomes from HPV16E7-pulsed DCs (see Figure 3C). Second, the ELISA data showed that exosomes 

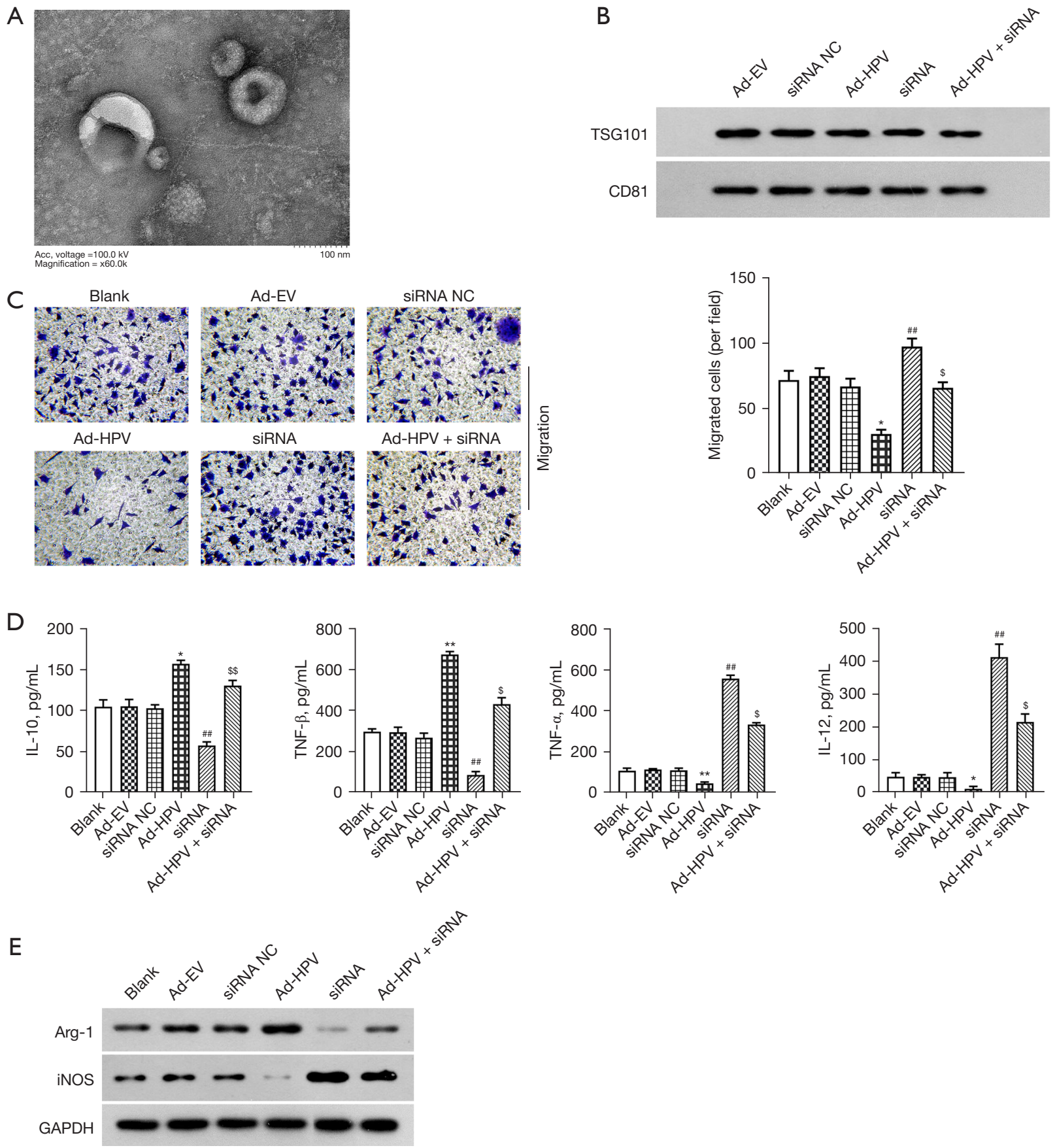

Figure 3 Exosomes from HPV16E7-pulsed or/and CAT2-silenced DCs regulated macrophage differentiation and migration. Exosomes were isolated from DCs that had overexpressed HPV16E7 and/or silenced CAT2, then these exosomes were applied to treat M0 macrophages. (A) The morphology of exosomes was first observed using a TEM. (B) Western blot displayed the expression change of the exosome marker proteins (TSG101 and CD81) in each group of exosomes. (C) Transwell assays were conducted by crystal violet staining to examine the changes of migration ability in each group of macrophages. Magnification, 200×. (D) ELISA kits were used to assess the levels of IL-10, TGF- $\beta$, TNF- $\alpha$, and IL-12 in each group of macrophages. (E) Arg-1 and iNOS expressions were identified by Western blot. *, $\mathrm{P}<0.05 ;{ }^{* *}, \mathrm{P}<0.01$ vs. Ad-EV; ${ }^{\#}, \mathrm{P}<0.01$ vs. siRNA NC; ${ }^{\$}, \mathrm{P}<0.05 ;{ }^{\$}, \mathrm{P}<0.01$ vs. siRNA. Ad-EV, adenovirus loaded with empty vector; Arg-1, arginase-1; CAT2, catalase 2; DCs, dendritic cells; HPV16E7, human papillomavirus type 16 E7; IL-10, interleukin-10; IL-12, interleukin-12; iNOS, inducible nitric oxide synthase; NC, negative control; TEM, transmission electron microscope; TGF- $\beta$, transforming growth factor beta; TNF- $\alpha$, tumor necrosis factor alpha. 
from HPV16E7-pulsed DCs significantly increased IL10 and TGF- $\beta$ levels, and significantly decreased TNF- $\alpha$ and IL-12 levels in macrophages, while exosomes from CAT2-silenced DCs significantly lowered IL-10 and TGF- $\beta$ levels, and significantly increased TNF- $\alpha$ and IL12 levels in macrophages, and exosomes from HPV16E7pulsed DCs and exosomes from CAT2-silenced DCs reversed the effect of the other party on IL-10, TGF- $\beta$, TNF- $\alpha$, and IL-12 levels in macrophages (see Figure 3D). Further, we found that exosomes from HPV16E7-pulsed DCs significantly upregulated Arg-1 and downregulated iNOS in macrophages, while exosomes from CAT2silenced DCs downregulated Arg-1 and upregulated iNOS in macrophages, and both also significantly attenuated the regulation of the other side on Arg-1 and iNOS expressions in macrophages (see Figure $3 E$ ). Thus, we found that exosomes from HPV16E7-pulsed DCs and exosomes from CAT2-silenced DCs affected the migration and polarization of macrophages.

\section{GW4869 treatment reversed the effects of HPV16E7- pulsed and CAT2-silenced DCs on macrophage differentiation and migration}

We then examined whether the effects of HPV16E7pulsed and/or CAT2-silenced DCs on the polarization and migration of macrophages could be achieved through exosomes secreted by these DCs. First, we treated HPV16E7-pulsed or/and CAT2-silenced DCs with exosome inhibitor (GW4869), and then used the treated DCs to co-culture M0 macrophages. As per the results of the Transwell experiment, HPV16E7-pulsed DCs significantly repressed the migration of macrophages, while CAT2-silenced DCs significantly reversed the inhibition of HPV16E7-pulsed DCs on macrophage migration, and GW4869 treatment also significantly reduced the effect of HPV16E7-pulsed and CAT2-silenced DCs on migration capacity of macrophages (see Figure 4A). Next, the ELISA data showed that HPV16E7-pulsed DCs significantly increased IL-10 and TGF- $\beta$ levels, and decreased TNF- $\alpha$ and IL-12 levels in macrophages, which were also significantly weakened by CAT2-silenced DCs. Further, we found that the introduction of GW4869 also altered the levels of IL-10, TGF- $\beta$, IL-12, and TNF- $\alpha$ mediated by HPV16E7-pulsed and CAT2-silenced DCs in macrophages (see Figure 4B). The Western blotting data revealed that HPV16E7-pulsed DCs significantly upregulated Arg1 and downregulated iNOS in macrophages, which were also significantly attenuated by CAT2-silenced DCs, and GW4869 also significantly reversed the effect of HPV16E7-pulsed and CAT2-silenced DCs onArg-1 and iNOS expressions (see Figure 4C). In short, the data further revealed that HPV16E7-pulsed and CAT2-silenced DCs affected the differentiation and migration of macrophage by secreting exosomes.

\section{Exosomes from CAT2-silenced DCs suppressed growth and macrophage differentiation in the mice xenograft model of HPV cervical cancer}

Through in vivo experiments, we further examined the effect of CAT2-silenced DCs on tumor growth and macrophage polarization in HPV cervical cancer mice. First, we detached exosomes from CAT2-silenced DCs in nude mice, and then injected these exosomes into the HPV cervical cancer mice. We found that the tumors of the mice in the DC group were larger than those of mice in the blank group, and that tumor growth mediated by DC was also blocked by exosomes from CAT2-silenced DCs in HPV cervical cancer mice (see Figure 5A-5C). Additionally, the Western blot results revealed that Arg-1 was more upregulated and iNOS was more downregulated in the DC-treated HPV cervical cancer mice than the blank group, while exosomes from CAT2-silenced DCs reduced Arg-1 expression and elevated iNOS in the DC-treated HPV cervical cancer mice (see Figure 5D). The real-time quantitative PCR (RTqPCR) results also revealed that the messenger RNA levels of Arg-1 and CAT2 were significantly more increased and the mRNA level of iNOS was significantly more decreased in the DC-treated HPV cervical cancer mice than the blank group, while exosomes from CAT2-silenced DCs downregulated Arg-1 and CAT2 and upregulated iNOS in the DC-treated HPV cervical cancer mice (see Figure 5E). Further, we found that the IL-10 and TGF- $\beta$ levels were significantly increased, and IL-12 and TNF- $\alpha$ levels were significantly decreased in the DC-treated HPV cervical cancer mice, and this effect could be reversed by exosomes from CAT2-silenced DCs (Figure 5F). Thus, we further confirmed that exosomes from CAT2silenced DCs prevented growth, polarized macrophages toward the M1 phenotype, and induced inflammation in the mice xenograft model of HPV cervical cancer.

\section{Discussion}

Persistent infection with HPV-16 can cause multiple 
A

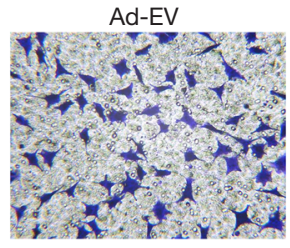

Ad-HPV + SiRNA

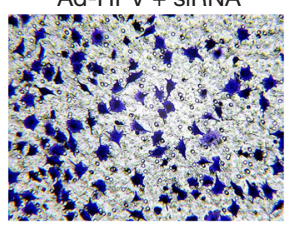

B

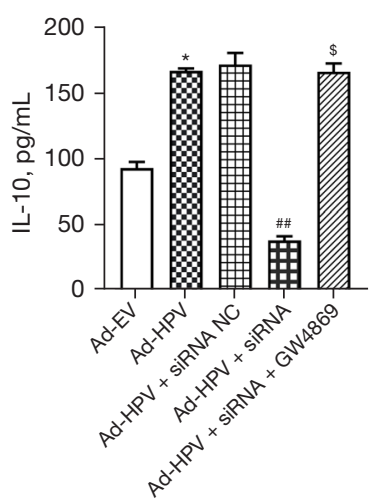

Ad-HPV

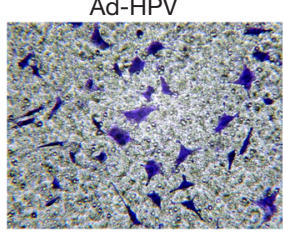

Ad-HPV + GW4869

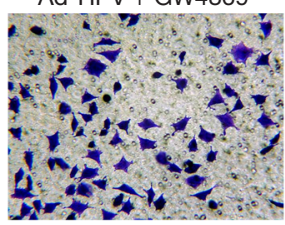

Ad-HPV + siRNA NC

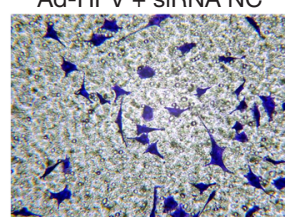

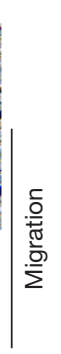
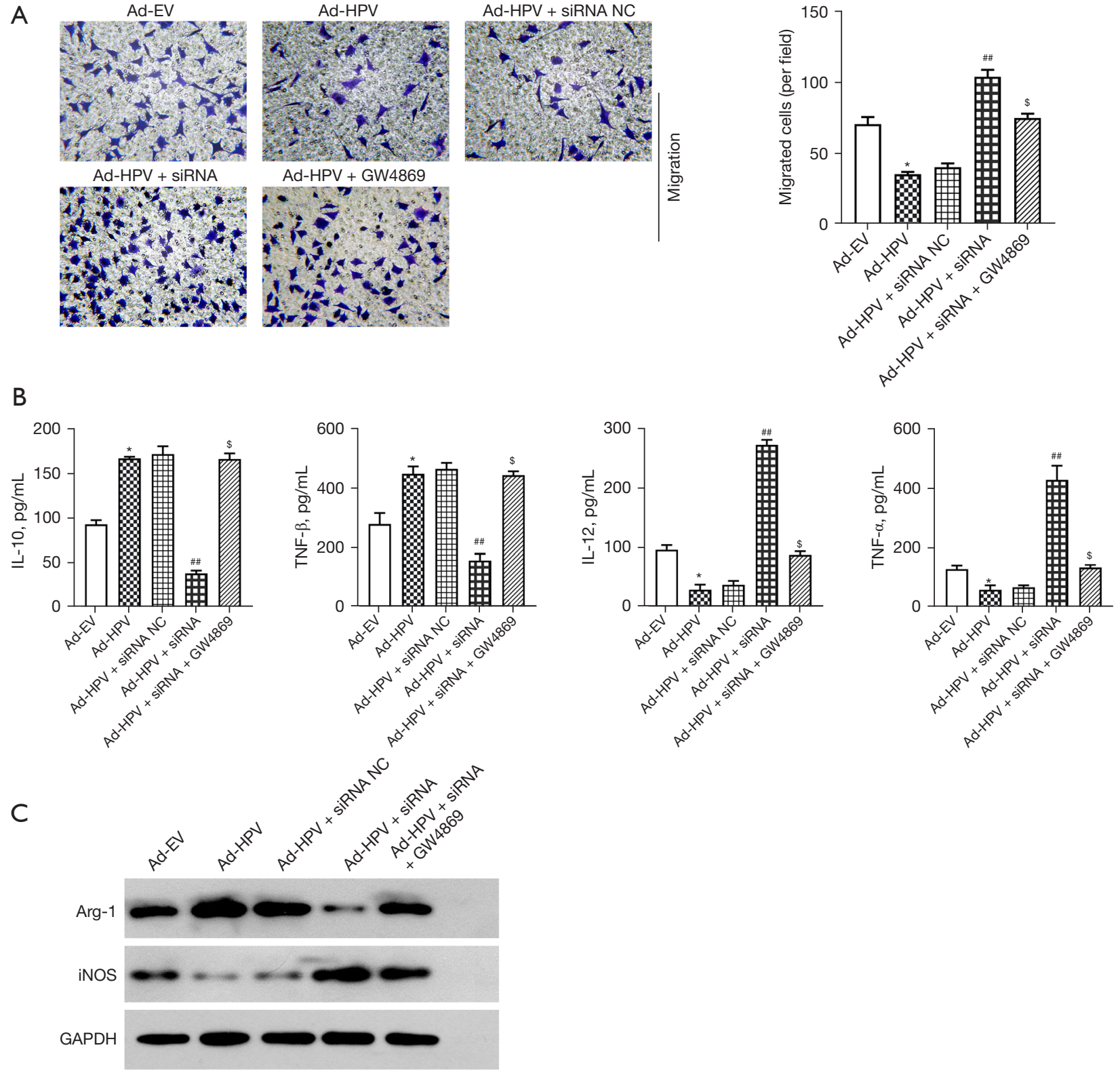

Figure 4 GW4869 treatment reversed the effects of HPV-16 E7-pulsed and CAT2-silenced DCs on macrophage differentiation and migration. HPV-16 E7-pulsed and/or CAT2-silenced DCs were treated with exosome inhibitor (GW4869), and then co-cultured with M0 macrophages. (A) Transwell assays were conducted by crystal violet staining to certify the migration capacity in the processed macrophages. Magnification, 200x. (B) ELISA kits were used to assess IL-10, TGF- $\beta$, IL-12 and TNF- $\alpha$ levels. (C) Western blot was applied to test Arg1 and iNOS expressions. *, $\mathrm{P}<0.05$ vs. Ad-EV; ${ }^{\#}, \mathrm{P}<0.01$ vs. Ad-HPV + siRNA NC; ${ }^{\$}, \mathrm{P}<0.05$ vs. Ad-HPV + siRNA. Ad-EV, adenovirus loaded with empty vector; Arg-1, arginase-1; CAT2, catalase 2; DCs, dendritic cells; HPV, human papillomavirus; IL-10, interleukin-10; IL12 , interleukin-12; iNOS, inducible nitric oxide synthase; NC, negative control; TGF- $\beta$, transforming growth factor beta; TNF- $\alpha$, tumor necrosis factor alpha.

cancers, most notably cervical cancer, which can express specific E6 and E7 proteins (6,27). The pathogenesis of cervical cancer is believed to be closely related to the early expression of E6 and E7 proteins (28). Notably, E7 protein can accelerate the independent growth and transformation of cells via the interaction of type 1 complement receptors 

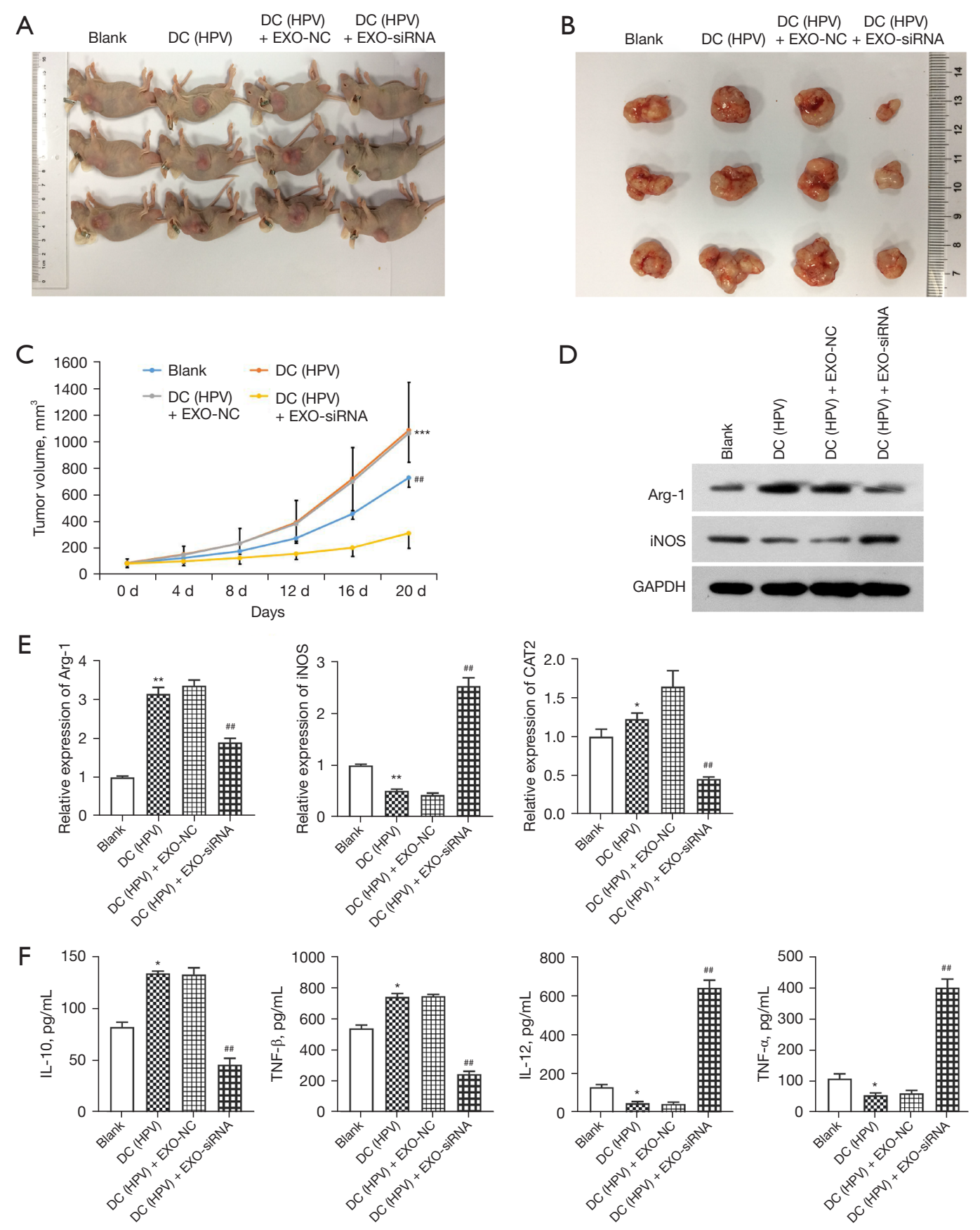

Figure 5 Exosomes from CAT2-silenced DCs suppressed growth and macrophage differentiation in the mice xenograft model of HPV cervical cancer. HPV cervical cancer mice were injected with exosomes, which were detached from CAT2-silenced DCs from nude mice. (A) Each group of nude mice was photographed and displayed. (B) Subcutaneous tumors were dissected and examined at the end of 4 weeks. (C) At days 7, 14, 21 and 28, we examined the tumor volume by measuring the length and width of the tumors of the nude mice. (D) Arg1 and iNOS expressions were evaluated by Western blot. (E) Arg-1, iNOS, and CAT2 expressions were confirmed via RT-qPCR. (F) IL-10, TGF- $\beta$, IL-12, and TNF- $\alpha$ levels were examined using ELISA kits. *, $\mathrm{P}<0.05 ;{ }^{* *}, \mathrm{P}<0.01$; ${ }^{* * *}, \mathrm{P}<0.001$ vs. Blank; ${ }^{* \#}, \mathrm{P}<0.01$ vs. DC (HPV) + EXO-NC. Arg-1, arginase-1; CAT2, catalase 2; DCs, dendritic cells; EXO, exosome; HPV, human papillomavirus; IL-10, interleukin-10; IL-12, interleukin-12; iNOS, inducible nitric oxide synthase; NC, negative control; TGF- $\beta$, transforming growth factor beta; TNF- $\alpha$, tumor necrosis factor alpha. 
and retinoblastoma, and then cause the transformation and immortalization of epithelial cells (29). Thus, tumor biotherapy based on $E 7$ gene has become a popular area of research.

Currently, the following 3 prophylactic HPV vaccines have been successfully marketed: gardasil, cervarix, and gardasil 9 (30). The prophylactic vaccines already on the market can protect against up to $90 \%$ of HPVassociated tumors (31). The vaccine carrying capsid antigen can stimulate a cellular immune response, but has no therapeutic effect in people already infected with HPV, as the basal epithelial cells carrying HPV can only express the early genes (32). To control the growth rate of cervical cancer patients as soon as possible, it is crucial that an HPV therapeutic vaccine be developed. DCs have been reported to have a strong capacity to intake and process antigens (33). DCs not only express the major histocompatibility complex (MHC)-I, MHC-II molecules, adhesion molecules (e.g., CD50, and CD54), and costimulatory molecules (e.g., CD40, MHC-II, and CD86), but also secrete cytokines, including IL-12, IL-6 and, TNF- $\alpha$ (34). DCs can also provide activation signals for $\mathrm{T}$ cells and induce the activation and proliferation of $\mathrm{T}$ cells (35). Thus, DC vaccines have great potential in generating anti-tumor immune responses.

In recent years, clinical trial of DC vaccines has been applied in multiple cancers, and have achieved some results (36). However, it needs further research to develop DC vaccines for different tumors. In this study, DCs from the peripheral blood of cervical cancer patients were transfected with HPV16E7 to obtain HPV16E7-pulsed DCs. We found that DC surface molecules (CD11c, CD80, CD83, and CD40) were highly expressed in the induced mDCs, which indicated the successful induction of DCs. Additionally, we found that that HPV16E7-pulsed DCs suppressed migration and induce M2 polarization in macrophages. However, the mechanism by which HPV16E7-pulsed DCs affect the function of macrophages has not been fully clarified.

Exosomes are nanoscale vesicles formed by the fusion of multivesicular bodies and cytoplasmic membranes, which can transfer information between cells (37). Exosomes are secreted by various living cells, and while exosomes share common characteristics, such as expressing CD9 and CD63, they also have their own unique characteristics (38). Notably, exosomes derived from DCs are not limited to acting by direct contact between cells, but can act on distant target cells through paracrine (39). It has been reported that DCderived exosomes can express MHC molecules, effectively activate $\mathrm{T}$ cells, induce antigen-specific $\mathrm{T}$ cell responses, and promote interferon (IFN)- $\gamma$ production $(40,41)$. Recent research has shown that DC-derived exosomes can induce an anti-tumor specific immune response in patients $(42,43)$. Thus, DC-derived exosomes are considered a beneficial substance secreted by DCs, which can produce immune stimulation (44). In our study, we successfully extracted and identified exosomes from HPV16E7-pulsed DCs, and first showed that exosomes from HPV16E7-pulsed DCs could block migration and M1 polarization in macrophages, and then showed that the inhibition of HPV16E7-pulsed DCs on the migration and M1 polarization of macrophages could also be achieved by secreting exosomes.

Immunosuppressive factors of the tumor microenvironment include myeloid-derived suppressor cells (MDSCs), TAMs, and regulatory $\mathrm{T}$ cells (45). Research has shown that MDSCs can mediate immunosuppression by different mechanisms, such as the secretion of immunosuppressive cytokines and the release of metabolites (46). During MDSC metabolism, the increased activity of Arg-1 and iNOS are markers of the inhibitory function of MDSCs, which requires L-arginine (L-Arg) consumption in the tumor microenvironment (47). Thus, L-Arg transport can regulate the immunosuppressive function of MDSCs (48). It has been reported that the y+ transport system is highly selective for cationic amino acids and is the main route by which L-Arg enters cells (49). When the demand for L-Arg increases, CAT2 expression is induced, and the rapid transport capacity of L-Arg is improved (50). Research has also confirmed that the suppression of CAT2 can help induce the inhibitory function of MDSCs and enhance the anti-tumor effect of $T$ cells (51). Thus, blocking CAT2 activity can reduce L-Arg transport, improve the tumor microenvironment and enhance anti-tumor immunity. In our study, we further verified that CAT2-silenced DCs accelerated the migration and $\mathrm{M} 1$ polarization of macrophages by secreting exosomes. Additionally, we showed that there is a significant reversal effect between HPV16E7-pulsed DCs and CAT2-silenced DCs in the migration and M1 polarization of macrophages. Moreover, exosomes from CAT2-silenced DCs also prevented growth and M2 polarization in the mice xenograft model of HPV cervical cancer.

\section{Conclusions}

Our current study verified that HPV16E7-pulsed DCs inhibited migration and $\mathrm{M} 1$ polarization in macrophages by secreting exosomes, and CAT2-silenced DCs had the opposite effect on HPV16E7-pulsed DCs in the migration 
and polarization of macrophages. Additionally, we found that the exosomes from HPV16E7-pulsed DCs and from CAT2-silenced DCs also affected macrophage migration and polarization. Thus, our current study has certain scientific significance for anti-tumor immunotherapy based on DCs.

\section{Acknowledgments}

Funding: This study was supported by funding from the Guangzhou Science and Technology Plan FoundationGeneral Project Topic (No. 201804010150), and the Guangzhou Science and Technology Plan FoundationApplication Foundation Project (No. 202102080622 and No. 202102080623).

\section{Footnote}

Reporting Checklist: The authors have completed the ARRIVE reporting checklist. Available at https://atm. amegroups.com/article/view/10.21037/atm-21-6998/rc

Data Sharing Statement: Available at https://atm.amegroups. com/article/view/10.21037/atm-21-6998/dss

Conflicts of Interest: All authors have completed the ICMJE uniform disclosure form (available at https://atm. amegroups.com/article/view/10.21037/atm-21-6998/coif). The authors have no conflicts of interest to declare.

Ethical Statement: The authors are accountable for all aspects of the work in ensuring that questions related to the accuracy or integrity of any part of the work are appropriately investigated and resolved. The study was approved by the Ethics Committee of the People's Hospital of Huadu District and conducted in accordance with the Declaration of Helsinki (as revised in 2013). Informed consent was granted an exemption by the hospital. All animal experiments were performed and approved by the Affiliated Huadu Hospital, Southern Medical University, in compliance with the Affiliated Huadu Hospital of Southern Medical University guidelines for the care and use of animals.

Open Access Statement: This is an Open Access article distributed in accordance with the Creative Commons Attribution-NonCommercial-NoDerivs 4.0 International License (CC BY-NC-ND 4.0), which permits the non- commercial replication and distribution of the article with the strict proviso that no changes or edits are made and the original work is properly cited (including links to both the formal publication through the relevant DOI and the license). See: https://creativecommons.org/licenses/by-nc-nd/4.0/.

\section{References}

1. $\mathrm{Vu} \mathrm{M}, \mathrm{Yu} \mathrm{J}$, Awolude OA, et al. Cervical cancer worldwide. Curr Probl Cancer 2018;42:457-65.

2. Ba DM, Ssentongo P, Musa J, et al. Prevalence and determinants of cervical cancer screening in five subSaharan African countries: A population-based study. Cancer Epidemiol 2021;72:101930.

3. Bedell SL, Goldstein LS, Goldstein AR, et al. Cervical Cancer Screening: Past, Present, and Future. Sex Med Rev 2020;8:28-37.

4. Kessler TA. Cervical Cancer: Prevention and Early Detection. Semin Oncol Nurs 2017;33:172-83.

5. Hu Z, Ma D. The precision prevention and therapy of HPV-related cervical cancer: new concepts and clinical implications. Cancer Med 2018;7:5217-36.

6. Feng Q, Li Q, Bao K, et al. Analysis and comparison of the prevalence and genotype distribution of human papillomavirus in two different areas of China: a crosssectional study. Eur J Gynaecol Oncol 2021;42:795-801.

7. Wang Q, Song R, Zhao C, et al. HPV16 E6 promotes cervical cancer cell migration and invasion by downregulation of NHERF1. Int J Cancer 2019;144:1619-32.

8. Jiang P, Wang L, Hou B, et al. A novel HPV16 E7affitoxin for targeted therapy of HPV16-induced human cervical cancer. Theranostics 2018;8:3544-58.

9. Collin M, Ginhoux F. Human dendritic cells. Semin Cell Dev Biol 2019;86:1-2.

10. Bryant CE, Sutherland S, Kong B, et al. Dendritic cells as cancer therapeutics. Semin Cell Dev Biol 2019;86:77-88.

11. Lopes AMM, Michelin MA, Murta EFC. Monocytederived dendritic cells from patients with cervical intraepithelial lesions. Oncol Lett 2017;13:1456-62.

12. Chen S, Lv M, Fang S, et al. Poly(I:C) enhanced anticervical cancer immunities induced by dendritic cellsderived exosomes. Int J Biol Macromol 2018;113:1182-7.

13. Gardner A, de Mingo Pulido Á, Ruffell B. Dendritic Cells and Their Role in Immunotherapy. Front Immunol 2020;11:924.

14. Gardner A, Ruffell B. Dendritic Cells and Cancer Immunity. Trends Immunol 2016;37:855-65.

15. Abraham RS, Mitchell DA. Gene-modified dendritic cell 
vaccines for cancer. Cytotherapy 2016;18:1446-55.

16. Perez S, Zimet GD, Tatar O, et al. Human Papillomavirus Vaccines: Successes and Future Challenges. Drugs 2018;78:1385-96.

17. Wang R, Pan W, Jin L, et al. Human papillomavirus vaccine against cervical cancer: Opportunity and challenge. Cancer Lett 2020;471:88-102.

18. Hancock G, Hellner K, Dorrell L. Therapeutic $\mathrm{HPV}$ vaccines. Best Pract Res Clin Obstet Gynaecol 2018;47:59-72.

19. Chabeda A, Yanez RJR, Lamprecht R, et al. Therapeutic vaccines for high-risk HPV-associated diseases. Papillomavirus Res 2018;5:46-58.

20. Bolhassani A, Shahbazi S, Agi E, et al. Modified DCs and MSCs with HPV E7 antigen and small Hsps: Which one is the most potent strategy for eradication of tumors? Mol Immunol 2019;108:102-10.

21. Bellone S, El-Sahwi K, Cocco E, et al. Human papillomavirus type 16 (HPV-16) virus-like particle L1specific CD8+ cytotoxic T lymphocytes (CTLs) are equally effective as E7-specific CD8+ CTLs in killing autologous $\mathrm{HPV}-16$-positive tumor cells in cervical cancer patients: implications for L1 dendritic cell-based therapeutic vaccines. J Virol 2009;83:6779-89.

22. Pan Y, Yu Y, Wang X, et al. Tumor-Associated Macrophages in Tumor Immunity. Front Immunol 2020;11:583084.

23. Wu K, Lin K, Li X, et al. Redefining Tumor-Associated Macrophage Subpopulations and Functions in the Tumor Microenvironment. Front Immunol 2020;11:1731.

24. Chen XJ, Wu S, Yan RM, et al. The role of the hypoxiaNrp-1 axis in the activation of M2-like tumor-associated macrophages in the tumor microenvironment of cervical cancer. Mol Carcinog 2019;58:388-97.

25. Liu Y, Li L, Li Y, et al. Research Progress on TumorAssociated Macrophages and Inflammation in Cervical Cancer. Biomed Res Int 2020;2020:6842963.

26. Sadeghzadeh M, Bornehdeli S, Mohahammadrezakhani H, et al. Dendritic cell therapy in cancer treatment; the stateof-the-art. Life Sci 2020;254:117580.

27. Balasubramaniam SD, Balakrishnan V, Oon CE, et al. Key Molecular Events in Cervical Cancer Development. Medicina (Kaunas) 2019;55:384.

28. Sato N, Saga Y, Uchibori R, et al. Eradication of cervical cancer in vivo by an AAV vector that encodes shRNA targeting human papillomavirus type $16 \mathrm{E} 6 / \mathrm{E} 7$. Int J Oncol 2018;52:687-96.

29. Noval MG, Gallo M, Perrone S, et al. Conformational dissection of a viral intrinsically disordered domain involved in cellular transformation. PLoS One 2013;8:e72760.

30. Cheng L, Wang Y, Du J. Human Papillomavirus Vaccines: An Updated Review. Vaccines (Basel) 2020;8:391.

31. Mlad nka A, Sláma J. Vaccination against HPV and view of new possibilities. Ceska Gynekol Winter;83:218-25.

32. Kunda NK, Peabody J, Zhai L, et al. Evaluation of the thermal stability and the protective efficacy of spray-dried HPV vaccine, Gardasil@ 9. Hum Vaccin Immunother 2019;15:1995-2002.

33. Hansen M, Andersen MH. The role of dendritic cells in cancer. Semin Immunopathol 2017;39:307-16.

34. Kohli K, Pillarisetty VG. Dendritic Cells in the Tumor Microenvironment. Adv Exp Med Biol 2020;1273:29-38.

35. Takenaka MC, Quintana FJ. Tolerogenic dendritic cells. Semin Immunopathol 2017;39:113-20.

36. Veglia F, Gabrilovich DI. Dendritic cells in cancer: the role revisited. Curr Opin Immunol 2017;45:43-51.

37. Lin J, Li J, Huang B, et al. Exosomes: novel biomarkers for clinical diagnosis. ScientificWorldJournal 2015;2015:657086.

38. Zhang L, Yu D. Exosomes in cancer development, metastasis, and immunity. Biochim Biophys Acta Rev Cancer 2019;1871:455-68.

39. Greening DW, Gopal SK, Xu R, et al. Exosomes and their roles in immune regulation and cancer. Semin Cell Dev Biol 2015;40:72-81.

40. Romagnoli GG, Zelante BB, Toniolo PA, et al. Dendritic Cell-Derived Exosomes may be a Tool for Cancer Immunotherapy by Converting Tumor Cells into Immunogenic Targets. Front Immunol 2014;5:692.

41. Liu H, Gao W, Yuan J, et al. Exosomes derived from dendritic cells improve cardiac function via activation of CD4(+) T lymphocytes after myocardial infarction. J Mol Cell Cardiol 2016;91:123-33.

42. Lu Z, Zuo B, Jing R, et al. Dendritic cell-derived exosomes elicit tumor regression in autochthonous hepatocellular carcinoma mouse models. J Hepatol 2017;67:739-48.

43. Pitt JM, André F, Amigorena S, et al. Dendritic cellderived exosomes for cancer therapy. J Clin Invest 2016;126:1224-32.

44. Tian H, Li W. Dendritic cell-derived exosomes for cancer immunotherapy: hope and challenges. Ann Transl Med 2017;5:221.

45. Davis RJ, Van Waes C, Allen CT. Overcoming barriers to effective immunotherapy: MDSCs, TAMs, and Tregs as mediators of the immunosuppressive microenvironment in 


\section{Page 14 of 14}

head and neck cancer. Oral Oncol 2016;58:59-70.

46. Yang F, Li Y, Zhang Q, et al. The Effect of Immunosuppressive Drugs on MDSCs in Transplantation. J Immunol Res 2018;2018:5414808.

47. Ren W, Zhang X, Li W, et al. Circulating and tumorinfiltrating arginase 1-expressing cells in gastric adenocarcinoma patients were mainly immature and monocytic Myeloid-derived suppressor cells. Sci Rep 2020;10:8056.

48. Cao Y, Feng Y, Zhang Y, et al. L-Arginine supplementation inhibits the growth of breast cancer by enhancing innate and adaptive immune responses mediated by suppression
Zhang et al. Exosomes from HPV16E7-pulsed DCs in cervical cancer

of MDSCs in vivo. BMC Cancer 2016;16:343.

49. Zakoji N, Akanuma S, Tachikawa M, et al. Involvement of cationic amino acid transporter 1 in L-arginine transport in rat retinal pericytes. Biol Pharm Bull 2015;38:257-62.

50. Nicholson B, Manner CK, MacLeod CL. Cat2 L-arginine transporter-deficient fibroblasts can sustain nitric oxide production. Nitric Oxide 2002;7:236-43.

51. Banjarnahor S, Rodionov RN, König J, et al. Transport of L-Arginine Related Cardiovascular Risk Markers. J Clin Med 2020;9:3975.

(English Language Editor: L. Huleatt)

Cite this article as: Zhang G, Liao Y, Pan X, Zhang X. Exosomes from HPV-16 E7-pulsed dendritic cells prevent the migration, $\mathrm{M} 1$ polarization, and inflammation of macrophages in cervical cancer by regulating catalase 2 (CAT2). Ann Transl Med 2022;10(4):217. doi: 10.21037/atm-21-6998 